\title{
On Optimal Link Activation with Interference Cancellation in Wireless Networking
}

Di Yuan, Vangelis Angelakis, Lei Chen, Eleftherios Karipidis and Erik G. Larsson

\section{Linköping University Post Print}

N.B.: When citing this work, cite the original article.

(C2012 IEEE. Personal use of this material is permitted. However, permission to reprint/republish this material for advertising or promotional purposes or for creating new collective works for resale or redistribution to servers or lists, or to reuse any copyrighted component of this work in other works must be obtained from the IEEE.

Di Yuan, Vangelis Angelakis, Lei Chen, Eleftherios Karipidis and Erik G. Larsson, On Optimal Link Activation with Interference Cancellation in Wireless Networking, 2012, accepted IEEE Transactions on Vehicular Technology.

Postprint available at: Linköping University Electronic Press

http://urn.kb.se/resolve?urn=urn:nbn:se:liu:diva-81933 


\title{
On Optimal Link Activation with Interference Cancellation in Wireless Networking
}

\author{
Di Yuan, Member, IEEE, Vangelis Angelakis, Member, IEEE, Lei Chen, Student Member, IEEE, \\ Eleftherios Karipidis, Member, IEEE, and Erik G. Larsson, Senior Member, IEEE
}

\begin{abstract}
A fundamental aspect in performance engineering of wireless networks is optimizing the set of links that can be concurrently activated to meet given signal-to-interference-andnoise ratio (SINR) thresholds. The solution of this combinatorial problem is the key element in scheduling and cross-layer resource management. In this paper, we assume multiuser decoding receivers, which can cancel strongly interfering signals. As a result, in contrast to classical spatial reuse, links being close to each other are more likely to be active concurrently. Our focus is to gauge the gain of successive interference cancellation (SIC), as well as the simpler, yet instructive, case of parallel interference cancellation (PIC), in the context of optimal link activation. We show that both problems are NP-hard and develop compact integer linear programming formulations that enable to approach global optimality. We provide an extensive numerical performance evaluation, indicating that for low to medium SINR thresholds the improvement is quite substantial, especially with SIC, whereas for high SINR thresholds the improvement diminishes and both schemes perform equally well.
\end{abstract}

\section{INTRODUCTION}

In wireless networking, determining the sets of links that can be active simultaneously is a cornerstone optimization task of combinatorial nature. For a link to be active, a given signal-to-interference-and-noise ratio (SINR) threshold must be met according to the physical model [12]. Most of previous analyses assume single-user decoding (SUD) receivers that treat interference as additive noise. For interference-limited scenarios, orthogonalization, aka scheduling, along some dimension of freedom (e.g., time), is necessary. The schedule is composed by link subsets, each being a solution to the link activation (LA) problem. Thus repeatedly performing LA is the dominant computational task in scheduling. Intuitively, with SUD, concurrently active links being spatially separated is preferable, as they generate little interference to each other,

Copyright (c) 2012 IEEE. Personal use of this material is permitted. However, permission to use this material for any other purposes must be obtained from the IEEE by sending a request to pubs-permissions@ieee.org.

D. Yuan, V. Angelakis, and L. Chen are with the Department of Science and Technology (ITN), Linköping University, SE-601 74 Norrköping, Sweden (e-mail: diyua@itn.liu.se,vanan@itn.liu.se, leich@itn.liu.se).

E. Karipidis and E. G. Larsson are with the Communication Systems Division, Department of Electrical Engineering (ISY), Linköping University, SE-581 83 Linköping, Sweden (e-mail: karipidis@isy.liu.se, erik.larsson@isy.liu.se).

This work has been supported in part by the Excellence Center at Linköping-Lund in Information Technology (ELLIIT), the Center for Industrial Information Technology (CENIIT) at Linköping University, and the Swedish Foundation for Strategic Research (SSF). This work has been performed in the framework of the European research project SAPHYRE, which is partly funded by the European Union under its FP7 ICT Objective 1.1 - The Network of the Future, and the EU-FP7 Marie Curie project FP7People-2007-3-1-IAPP-218309. and scheduling amounts to achieving optimal spatial reuse. Optimal LA has attracted a considerable amount of attention. See, for example, [1], [6], [8], [11], [24] for complexity, solution approximation, and exact algorithms. For scheduling and cross-layer resource management, we refer to [4], [7], [9], [17], [19] and the references therein.

We consider LA where each link is associated with a positive weight, with the objective of maximizing the total weight of active links. The weights represent utility, queue size [21], or dual prices in the column generation method for scheduling [4]. A special case is maximum-cardinality LA, for which all weights equal one. We consider optimal LA under the setup of multiuser decoding (MUD) capability [22]. Unlike noise, interference contains encoded information. This is exploited by MUD receivers to perform interference cancellation (IC). That is, the receivers, before decoding the signal of interest, first decode the interfering signals they are able to and remove them from the composite signal. For IC, a receiver acts as if it is the intended receiver of the interfering signal. An interfering signal can be cancelled, only if it is received with enough power in relation to the other transmissions, including the receiver's own signal of interest. In other words, the "interference-to-other-signals-and-noise" ratio (which is an intuitive but non-rigorous term) must meet the SINR threshold of the interfering signal. With MUD, concurrent activation of strongly interfering links becomes more likely, enabling activation patterns that are counter-intuitive in the conventional setting. Our focus is on the potential of IC in boosting the performance of LA. Because LA is a key element in resource management, the study opens up new perspectives of these problems as well. For our work, we assume that MUD is carried out without any significant performance impairments, hence our results effectively constitute bounds on what is achievable in practice.

In the multi-link setup, the optimal IC scheme is to allow every receiver to perform cancellations successively in multiple stages. In each stage, the receiver decodes and removes one interfering signal. This scheme is referred to as successive IC (SIC). From an optimization standpoint, modeling SIC is challenging, because the order of cancellations is of significance. Clearly, enumerating the potential cancellation orders will not scale at all. Thus compact formulations are essential. A simplified IC scheme is to consider only the cancellations that can be performed concurrently in a single stage, that is, when determining the possibility for the cancellation of an interfering link, all remaining transmissions, no matter whether or not they are also being examined for cancellation, are 
regarded as interference. We refer to this scheme as parallel IC (PIC). As some of the cancellations in SIC may not be possible in PIC, one can expect that the gain of the latter is less than that of the former. A further restriction is to allow at most one cancellation per receiver. This scheme, which we refer to as single-link IC (SLIC), poses additional limits on the performance. However, it is the simplest scheme for practical implementation. In comparison to SIC, PIC and SLIC are much easier to formulate mathematically, as ordering is not relevant. In this paper, PIC refers to concurrent decoding of as many interfering signals possible in a single stage, before the signal of interest is decoded. Thus, it is different from the PIC scheme in the context of CDMA multiuser receivers, which decodes all signals and requires multiple stages, canceling the interference of all signals simultaneously at each decision stage [22].

There is a growing amount of interest in exploiting the potential of IC. In [2], [15], we applied rate adaptation to exploit the benefits of SLIC and PIC in improving aggregate system throughput, and assessed the potential of SLIC for SINR balancing, respectively. In [16], the performance of SIC in aggregate throughput is evaluated for CDMA within a specific distributed scheduling protocol. In [14], the authors developed a framework for joint routing and SIC-enabled scheduling where all links have a common SINR threshold, and provided a numerical study on one multi-hop wireless network instance. In parallel, another contribution was made in [18], where the authors considered SIC-enabled system under the physical SINR model and introduced a greedy algorithm to construct schedules of bounded length.

In this correspondence, we formalize optimal LA with PIC and SIC, focusing on the latter, for both uniform and nonuniform SINR thresholds. An NP-hardness result is provided. We develop compact integer linear programming (ILP) formulations to approach global optimality for problem sizes of practical interest. We present representative results from an extensive numerical performance evaluation, providing insight into the attainable gains of adopting IC.

\section{Problem Definition And Complexity}

Consider a wireless system of $K$ pairs of transmitters and receivers, forming $K$ directed links. Let $\mathcal{K} \triangleq\{1, \ldots, K\}$. For $m, k \in \mathcal{K}, G_{m k}$ denotes the channel gain between $m$ 's transmitter and $k$ 's receiver. The noise power is denoted by $\eta$. For each $k \in \mathcal{K}$, the transmit power, SINR threshold, and weight are denoted by $p_{k}, \gamma_{k}$, and $w_{k}$, respectively. We consider first LA with SUD, denoted by LA-SUD, and define this baseline problem: Find an activation set $\mathcal{A} \subseteq \mathcal{K}$, maximizing $\sum_{k \in \mathcal{A}} w_{k}$ and satisfying the conditions $p_{k} G_{k k} /\left(\sum_{m \in \mathcal{A} \backslash\{k\}} p_{m} G_{m k}+\right.$ $\eta) \geq \gamma_{k}, \forall k \in \mathcal{A}$, which ensure that all active links meet the SINR thresholds.

LA-SUD can be represented by an ILP formulation; see, e.g., [8]. A set of binary variables $x_{k}, \forall k \in \mathcal{K}$, is used to indicate link activation. In order to ease comparisons to the formulations that are introduced later, we reproduce below the formulation of LA-SUD:

$$
\begin{array}{ll}
\max _{\left\{x_{k} \in\{0,1\}\right\}_{k \in \mathcal{K}}} \sum_{k \in \mathcal{K}} w_{k} x_{k} \\
\text { s. t. } & \frac{p_{k} G_{k k}+M_{k}\left(1-x_{k}\right)}{\sum_{m \neq k} p_{m} G_{m k} x_{m}+\eta} \geq \gamma_{k} \quad \forall k \in \mathcal{K} .
\end{array}
$$

The objective of maximizing the total weight is given in (1a). By (1b), if $x_{k}=1$, indicating that link $k$ is active, the SINR of link $k$ must be at least $\gamma_{k}$. If $x_{k}=0$, (1b) has null effect, if parameter $M_{k}$ is sufficiently large (e.g., $M_{k}=$ $\left.\sum_{m \neq k} p_{m} G_{m k} \gamma_{k}+\eta \gamma_{k}-p_{k} G_{k k}\right)$. In formulation (1), the numbers of variables and constraints are both of $O(K)$.

Next, we consider LA with MUD receivers, and define LA with PIC and SIC, denoted by LA-PIC and LA-SIC, respectively. Here, the definitions are formalized in a general style using sets and bijections. Developing compact and effective integer formulations of LA-PIC and LA-SIC, similar to the style of (1), form the bulk of Section III and IV, respectively.

For LA-PIC, the output includes sets of cancellations, in addition to the activation set $\mathcal{A}$. A formal definition is: Find an activation set $\mathcal{A} \subseteq \mathcal{K}$ and sets $\mathcal{C}_{k} \subseteq \mathcal{A} \backslash\{k\}$ of cancelled transmissions for each $k \in \mathcal{A}$, maximizing $\sum_{k \in \mathcal{A}} w_{k}$ and satisfying the conditions:

$$
\text { 1) } p_{m} G_{m k} /\left(\sum_{n \in \mathcal{A} \backslash\{m\}} p_{n} G_{n k}+\eta\right) \geq \gamma_{m}, \forall m \in \mathcal{C}_{k}, \forall k \in
$$$$
\text { 2) } p_{k} G_{k k} /\left(\sum_{m \in \mathcal{A} \backslash\left\{k, \mathcal{C}_{k}\right\}} p_{m} G_{m k}+\eta\right) \geq \gamma_{k}, \forall k \in \mathcal{A} \text {. }
$$

The first condition ensures that the specified cancellations can take place. If link $k$ 's receiver cancels $m$ 's transmission, the "interference-to-other-signals-and-noise" ratio must be at least $\gamma_{m}$. The second set of conditions formulates the SINR requirements for the signals of interest, and the cancelled terms do not appear in the denominator of SINR.

For SIC, the output must be further augmented in order to specify, for each $\mathcal{C}_{k}, k \in \mathcal{A}$, the order in which the cancellations take place. A formal definition of LA-SIC is: Find an activation set $\mathcal{A} \subseteq \mathcal{K}$ and sets $\mathcal{C}_{k} \subseteq \mathcal{A} \backslash\{k\}$ of cancelled transmissions, along with a bijection $b_{k}: \mathcal{C}_{k} \mapsto\left\{1, \ldots,\left|\mathcal{C}_{k}\right|\right\}$ for each $k \in \mathcal{A}$, maximizing $\sum_{k \in \mathcal{A}} w_{k}$ and satisfying the conditions:

1) $p_{m} G_{m k} /\left(\sum_{n \in \mathcal{A} \backslash\left\{m, q \in \mathcal{C}_{k}: b_{k}(q)<b_{k}(m)\right\}} p_{n} G_{n k}+\eta\right) \geq$ $\gamma_{m} \forall m \in \mathcal{C}_{k}, \forall k \in \mathcal{A}$;

2) $p_{k} G_{k k} /\left(\sum_{m \in \mathcal{A} \backslash\left\{k, \mathcal{C}_{k}\right\}} p_{m} G_{m k}+\eta\right) \geq \gamma_{k}, \forall k \in \mathcal{A}$.

The cancellation sequence for each active $k$ is given by the bijection $b_{k}$ that defines a unique mapping of the links in $\mathcal{C}_{k}$ to the IC order numbers in the sequence. That is, $b_{k}(m), m \in$ $\mathcal{C}_{k}$ is the stage at which $m$ is cancelled by the receiver of $k$. The bijection is used in the first condition to exclude the interference terms that have been cancelled in stages prior to $m$.

The baseline problem, LA-SUD, is known to be NP-hard; see, e.g., [11]. We provide the theoretical result that LA-PIC and LA-SIC remain NP-hard using a unified proof.

Theorem 1: Both LA-PIC and LA-SIC are NP-hard, even if the links both have uniform received powers at the receivers and uniform SINR thresholds.

Proof: LA-PIC and LA-SIC are clearly in NP. The proof applies a polynomial-time reduction from the maximum 
cardinality independent set (MIS) problem in a graph, of which we use $\mathcal{V}$ and $\mathcal{E}$ to denote the sets of vertexes and edges, respectively. In the reduction, we set $\mathcal{K}=\mathcal{V}$, and denote by $p^{\prime}$ the uniform received power on the $K$ links. Next, either the $K$ channel gain values or the transmit power values are chosen, as one is induced by the other. In either case, $p_{k}>p^{\prime}, k \in \mathcal{K}$. The SINR threshold $\gamma \triangleq 1$. For each edge $(m, k) \in \mathcal{E}$, we set $G_{k m} \triangleq \frac{p^{\prime}}{p_{k}}$ and $G_{m k} \triangleq \frac{p^{\prime}}{p_{m}}$ for links $m$ and $k$ in the LA instance. For any pair $m^{\prime}$ and $k^{\prime}$ of the remaining links, $G_{k^{\prime} m^{\prime}} \triangleq \frac{p^{\prime}}{K p_{k^{\prime}}}$ and $G_{m^{\prime} k^{\prime}} \triangleq \frac{p^{\prime}}{K p_{m^{\prime}}}$. Finally, $w_{k}=1, \forall k \in \mathcal{K}$, and $\eta \triangleq \frac{p^{\prime}}{K}$.

It follows from the construction that, even though IC is allowed, no cancellation takes place, as the decoding ratio for $m$ 's signal at $k$ 's receiver is at most $p^{\prime} /\left(p^{\prime}+\frac{p^{\prime}}{K}\right)<1$. Thus for the given scenario, LA-PIC and LA-SIC coincide with LA-SUD. In addition, for any two links with an edge in the graph, at most one can be active, because the SINR drops below $p^{\prime} /\left(p^{\prime}+\frac{p^{\prime}}{K}\right)<1$ if both transmit. On the other hand, for any $k \in \mathcal{K}$, its activation is not constrained by links that do not form edges with $k$ in the graph, as the SINR is at least $p^{\prime} /\left(\frac{K-1}{K} p^{\prime}+\frac{1}{K} p^{\prime}\right)=1$. Hence LA is equivalent to MIS, and the theorem follows.

The proof leads to three observations. First, LA-PIC and LA-SIC are hard to approximate - by the result of Håstad [13], there is no $\frac{1}{K^{1-\epsilon}}$ approximation for any $\epsilon>0$ for clique (which is equivalent to MIS in the complementary graph), unless $\mathrm{P}=$ NP. Second, the proof holds also for any $\gamma>1$, indicating that IC is not likely for large SINR thresholds. Third, if $\gamma$ is very small, IC will take place maximally, such that all links can be active simultaneously. In this case, LA-PIC and LA-SIC are trivially solved, whereas LA-SUD remains NP-hard as it is still equivalent to MIS. The observation justifies the above study of complexity.

\section{Link ACTIVATION With PARALLEL INTERFERENCE CANCELLATION}

We provide a compact ILP formulation for LA-PIC below. In addition to the $x$-variables, we introduce a second set of binary variables, $y_{m k}$, that is one if the receiver of link $k$ decodes and cancels the interference from link $m$ and zero otherwise, $\forall m, k \in \mathcal{K}, m \neq k$.

$$
\begin{array}{ll} 
& \max _{\left\{x_{k} \in\{0,1\}\right\}_{k \in \mathcal{K}}} \sum_{k \in \mathcal{K}} w_{k} x_{k} \\
\text { s. t. } & y_{m k} \in\{0,1\} \quad \forall m, k \in \mathcal{K}, m \neq k, \\
& y_{m k} \leq x_{m} \quad \forall m, k \in \mathcal{K}, m \neq k, \\
& y_{m k} \leq x_{k} \quad \forall m, k \in \mathcal{K}, m \neq k, \\
& \frac{p_{k} G_{k k}+M_{k}\left(1-x_{k}\right)}{\sum_{m \neq k} p_{m} G_{m k}\left(x_{m}-y_{m k}\right)+\eta} \geq \gamma_{k} \quad \forall k \in \mathcal{K}, \\
& \frac{p_{m} G_{m k}+M_{m k}\left(1-y_{m k}\right)}{\sum_{n \neq m} p_{n} G_{n k} x_{n}+\eta} \geq \gamma_{m} \\
& \forall m, k \in \mathcal{K}, m \neq k .
\end{array}
$$

Inequalities (2c) and (2d) state that a cancellation can take place $\left(y_{m k}=1\right)$, only if both $k$ and $m$ are active $\left(x_{k}=\right.$ $\left.x_{m}=1\right)$. Inequalities $(2 \mathrm{e})$ are SINR requirements similar to (1b) for LA-SUD, with the difference that IC is accounted for via the term $x_{m}-y_{m k}$. Note that the formulation will fail without $(2 \mathrm{c})$, because then it is possible to reduce the righthand side of $(2 \mathrm{e})$ by subtracting non-existing interference of non-active links. Constraints (2f) formulate the PIC condition: $y_{m k}$ can be one only if the interference from $m, p_{m} G_{m k}$, in relation to all other signals, including the signal of interest and transmissions being cancelled in parallel, meets $\gamma_{m}$. Setting $y_{m k}=0$ is always feasible, provided that the parameter $M_{m k}$ is large enough (e.g., $M_{m k} \triangleq \sum_{n \neq m} p_{n} G_{n k} \gamma_{m}+\eta \gamma_{m}-$ $\left.p_{m} G_{m k}\right)$. The size of (2), both in the numbers of variables and constraints, is of $O\left(K^{2}\right)$, which is one magnitude larger than that of (1); in fact, to incorporate IC between link pairs, one cannot expect a formulation of smaller size. While solving (2), two pre-processing steps can be applied. First, SNR-infeasible links are deleted, i.e., $x_{k}=0$ if $\frac{p_{k} G_{k k}}{\eta}<\gamma_{k}$. Second, if $\frac{p_{m} G_{m k}}{p_{k} G_{k k}+\eta}<\gamma_{m}$, then cancellation cannot be performed to $m$ at $k$ 's receiver, and corresponding $y$-variable can be set to zero and discarded.

Cancelling at most one interfering signal is more practical to implement than PIC and SIC. The resulting LA problem, denoted LA-SLIC, is formulated by adding $\sum_{m \neq k} y_{m k} \leq 1$, $\forall k \in \mathcal{K}$, to (2). Note that, if $\gamma_{k} \geq 1, \forall k \in \mathcal{K}$, SLIC is equivalent to PIC, since any signal except the strongest one would have an SINR of less than one.

\section{Link ACtivation with Successive Interference CANCEllation}

Formulating LA-SIC by compact ILPs is challenging, because cancellation sequences have to be accounted for. We present our ILP approach in two steps for LA-SIC with uniform and non-uniform SINR thresholds, respectively. For the former, it is known that SIC should be performed in the order of descending received powers (e.g., [14]). That the order holds at global optimum of LA-SIC with a common $\gamma$ can be easily derived. Suppose $p_{m} G_{m k}>p_{n} G_{n k}$, but link $k$ cancels $n$ 's signal first, before canceling that of $m$. Then $p_{m} G_{m k}$ is part of the remaining interference while canceling $n$ 's signal as well as the other signals cancelled before decoding $m$ 's transmission. Consider decoding the signal of $m$ immediately before $n$ instead. Because $\gamma_{m}=\gamma_{n}=\gamma$ and $m$ gives stronger received power, $m$ 's signal can be decoded successfully. After canceling $m$ 's signal, the remaining cancellations obviously remain feasible. Therefore decoding in descending order of receiver powers is indeed optimal.

Based on the above result, for each link $k$, we sort the remaining links in descending order of received power values (as if they are all active) at $k$ 's receiver. The result is denoted by bijection $i_{k}: \mathcal{K} \backslash\{k\} \mapsto\{1, \ldots, K-1\}$, where $i_{k}(m)$ is the position of link $m$ in the sorted sequence. Let $c_{m k} \triangleq K-1-i_{k}(m)$ denote the number of links after $m$ in the sorted sequence for $k$. When considering the cancellation condition of interfering link $m$, interference can only originate from links appearing after $m$ in the sorted sequence of $k$. 
Below we present the resulting ILP for LA-SIC under common SINR threshold.

$$
\begin{aligned}
& \max _{\left\{x_{k} \in\{0,1\}\right\}_{k \in \mathcal{K}}} \sum_{k \in \mathcal{K}} w_{k} x_{k} \\
& \text { s. t. } \quad(2 \mathrm{~b}),(2 \mathrm{c}),(2 \mathrm{~d}),(2 \mathrm{e}), \\
& \frac{p_{m} G_{m k}+M_{m k}\left(1-y_{m k}\right)}{\sum_{n \neq k, i_{k}(n)>i_{k}(m)} p_{n} G_{n k} x_{n}+p_{k} G_{k k}+\eta} \geq \gamma \\
& \sum_{n \neq k, i_{k}(n)>i_{k}(m)} y_{n k} \leq c_{m k}\left(1-x_{m}+y_{m k}\right) \\
& \forall m, k \in \mathcal{K}, m \neq k, m \neq k .
\end{aligned}
$$

The above formulation reuses the variable definition and some of the constraints in the LA-PIC formulation (2). The constraint set (3b) specifies the SIC condition, making use of the aforementioned sorted sequences: While canceling the signal of $m$ at receiver $k$ in stage $i_{k}(m)$, the remaining interference is limited to the transmissions coming after $m$ in the sorted sequence of $k$, since all other active links giving higher received power have already been cancelled. The formulation is however not complete without (3c). These constraints ensure that the optimal decoding order is indeed followed. That is, if both $m$ and $n$ are active with $p_{m} G_{m k}>p_{n} G_{n k}$, and $n$ is cancelled by $k$, then $m$ must be cancelled by $k$ as well. Equivalently speaking, if $m$ is active but not cancelled by $k$, then none of the other links after $m$ in the sequence of $k$ may be cancelled. The constraint has no effect as long as $x_{m}=y_{m k}$. If link $m$ is active but not cancelled, $\left(x_{m}=1\right.$ and $\left.y_{m k}=0\right)$, the right-hand side becomes zero, and therefore no cancellation may occur for any $n$ with a position after $m$ in the ordered sequence. Note that the case $x_{m}=0$ but $y_{m k}=1$ cannot occur because of $(2 \mathrm{c})$.

The size of (3) is of $O\left(K^{2}\right)$. Thus for a common SINR threshold, LA-SIC can be formulated as compactly as LAPIC. We remark that similar pre-processing steps for (2) can be applied to reduce the size of (3).

With link-individual SINR thresholds, pre-ordering IC sequences does not apply. To see the point, consider link $k$ and two interfering links $m$ and $n$, with $p_{k} G_{k k}+\eta=0.5$. No other transmission is present. For link $n$, suppose $p_{n} G_{n k}=2$ and $\gamma_{n}=2$. Consider two cases for link $m$, for both $p_{n} G_{n k}>p_{m} G_{m k}$ holds: $p_{m} G_{m k}=0.5$ and $\gamma_{m}=1 / 4$, and $p_{m} G_{m k}=1$ and $\gamma_{m}=1 / 3$, respectively. In case one, both transmissions can be cancelled only if cancellation applies to $n$ first. However, the opposite order must be used for the second case.

To deal with individual SINR thresholds, our ILP approach uses for each pair of links, $m, k \in \mathcal{K}$, a set of binary variables $y_{m k}^{t}$, and represents the cancellation stage by $t \in[1, K-1]$. The range can be reduced to restrict the maximum number of cancellations for practical considerations. To this end, we define, for each $k \in \mathcal{K}$, an integer parameter $T_{k} \leq K-1$ and a set $\mathcal{T}_{k} \triangleq\left\{1, \ldots, T_{k}\right\}$. The ILP formulation of the general LA-SIC problem is given below.

$$
\begin{aligned}
& \max _{\left\{x_{k} \in\{0,1\}\right\}_{k \in \mathcal{K}}} \sum_{k \in \mathcal{K}} w_{k} x_{k} \\
& \text { s. t. } y_{m k}^{t} \in\{0,1\} \quad \forall m, k \in \mathcal{K}, m \neq k, \forall t \in \mathcal{T}_{k} \text {, } \\
& \sum_{t=1}^{T_{k}} y_{m k}^{t} \leq x_{m} \quad \forall m, k \in \mathcal{K}, m \neq k, \\
& \sum_{m \neq k} y_{m k}^{t} \leq x_{k} \quad \forall k \in \mathcal{K}, \forall t \in \mathcal{T}_{k}, \\
& \frac{p_{k} G_{k k}+M_{k}\left(1-x_{k}\right)}{\sum_{m \neq k} p_{m} G_{m k}\left(x_{m}-\sum_{t=1}^{T_{k}} y_{m k}^{t}\right)+\eta} \geq \gamma_{k} \quad \forall k \in \mathcal{K} \\
& p_{m} G_{m k}+M_{m k}\left(1-y_{m k}^{t}\right)
\end{aligned}
$$

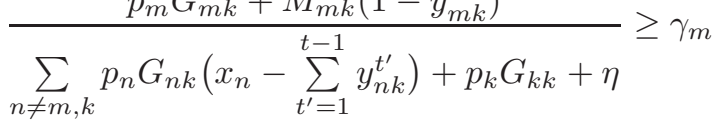

$$
\begin{aligned}
& \forall m, k \in \mathcal{K}, m \neq k, \forall t \in \mathcal{T}_{k}, \\
& \sum_{m \neq k} y_{m k}^{t} \leq \sum_{m \neq k} y_{m k}^{t-1} \quad \forall k \in \mathcal{K}, \quad \forall t \in \mathcal{T}_{k} \backslash\{1\} .(4
\end{aligned}
$$

The conditions $(4 c)-(4 d)$ have similar role with $(2 c)-(2 d)$. The summation over $t$ in (4c) and over $m$ in (4d) ensure, respectively, that a link is cancelled in at most one stage, and each receiver performs at most one cancellation per stage. The SINR requirements for decoding the signals of interest are set in (4e), in which all cancelled transmissions are excluded by means of subtraction in the right-hand side. Similarly, if link $m$ cancels the interference of link $k$ in stage $t$, the active interfering transmissions that have been cancelled before stage $t$ are excluded from the sum of undecoded interference, as stated in (4f), with the convention that the sum within the inner parenthesis is zero for $t=1$. The constraints $(4 \mathrm{~g})$ are not mandatory for the formulation's correctness; their role is to enhance the computational efficiency. These constraints ensure that the cancellations are performed as "early" as possible, i.e., there are no "idle" stages between stages where cancellation takes place.

The size of (4) is one magnitude larger than (3), as the numbers of variables and constraints are of $O\left(K^{3}\right)$. However, we note that the formulation (4) remains compact. In order to deal with the scalability issue, one may resort to a small value of $T_{k}$. Typically, doing so has little impact on the solution quality, because most of the performance gain from IC is due to the first few cancellations. Also, when implementing (4), pre-processing steps similar to those for (3) can be applied for size reduction.

\section{NumericAl RESUlts}

In this section, the ILP formulations proposed in Sections III-IV are utilized to numerically evaluate the performance gain due to single-link, parallel, and successive IC schemes over the baseline approach without IC. Two datasets are generated by uniformly scattering nodes in square areas of $500 \mathrm{~m} \times$ $500 \mathrm{~m}$. Dataset I takes an information-theoretic viewpoint [5], by arbitrarily choosing transmitter-receiver matchings with the sole criterion of feasible single-link activation. Dataset $\mathrm{N}$ 
30 links; I dataset; 30 instances

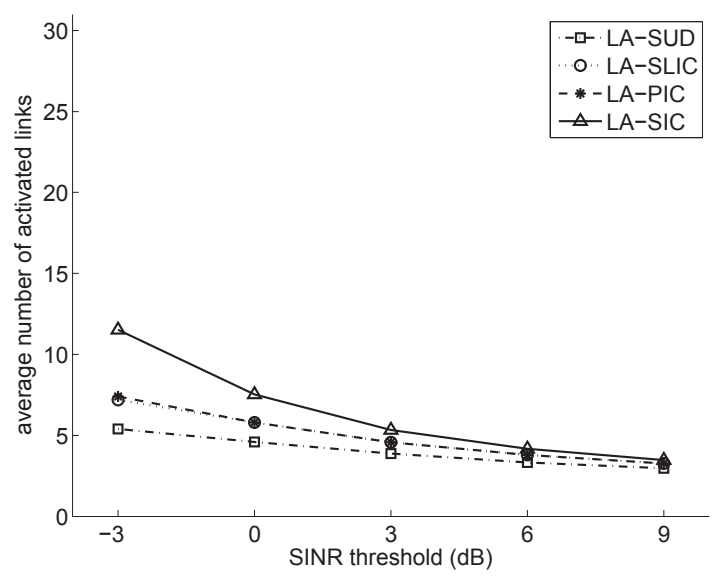

(a) I dataset

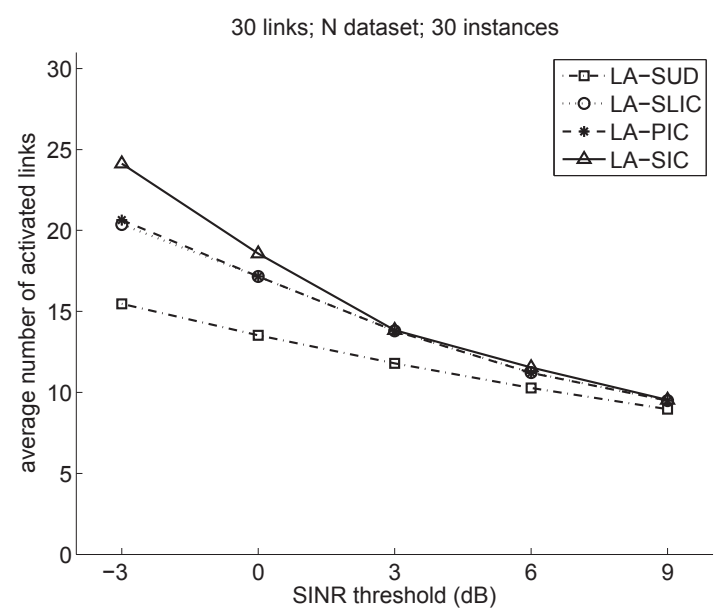

(b) $\mathrm{N}$ dataset

Fig. 1. Average number of activated links versus SINR threshold for a network of 30 links

resembles a multihop network [10], [20], by constraining the length of the links to be from $3 \mathrm{~m}$ up to $200 \mathrm{~m}$. The cardinality $K$ ranges from 5 up to 30 links. The input is chosen to be common for all links; specifically, $p_{k}=30 \mathrm{dBm}, \forall k \in \mathcal{K}$ and $\eta=-100 \mathrm{dBm}$. The channel gains $G_{m k}$ follow a geometric, distance-based, path loss model with an exponent of 4; hence, the length effectively determines the link SNR. Dataset I has minimum SNR of $16 \mathrm{~dB}$ and the mass of the SNR distribution is for $20-50 \mathrm{~dB}$, whereas dataset $\mathrm{N}$ has minimum SNR of $32 \mathrm{~dB}$ and the mass is for $40-60 \mathrm{~dB}$. For each dataset and network cardinality, the results presented are averages over 30 instances. Two simulation studies are conducted for the cases of LA with common and individual SINR thresholds, respectively.

At first, $\gamma$ takes values from $-3 \mathrm{~dB}$ up to $9 \mathrm{~dB}$ and all links have equal activation weights, e.g., $w_{k}=1, \forall k \in \mathcal{K}$. Fig. 1 compares, for networks of 30 links, the average performance of all LA schemes for various SINR thresholds; the left and right sub-figures correspond to the I and $\mathrm{N}$ datasets respectively. It is seen that the performance gains due to IC are significant, especially when the SINR threshold is low, but they diminish when the SINR threshold goes beyond 6dB. As expected, the performance increases with problem sophistication. Also, a comparison of Figs. 1a and $1 \mathrm{~b}$ shows that LA is easier for dataset N, even without IC due to the higher link SNRs. Nevertheless, the relative gain of SIC is more prominent in the case of the I dataset; specifically, for low SINR threshold SIC activates double number of links than SUD. For both datasets, SIC has an exponentially decreasing performance and for SINR thresholds up to about $3 \mathrm{~dB}$ it still significantly outperforms SUD. On the other hand, for SINR thresholds up to about $0 \mathrm{~dB}$, PIC yields a relatively constant performance improvement of roughly two to five links, for datasets I and $\mathrm{N}$ respectively. The performance of all IC schemes practically converges at an SINR threshold of $6 \mathrm{~dB}$. The interpretation is that if IC is possible, it is more likely that it will be restricted to a single link. For high SINR thresholds, above 6dB, it becomes less possible to perform IC. Also, as was discussed earlier in Section III, PIC and SLIC have identical performance for $\gamma \geq 1$. This is verified by the figure.

Next, the simulation results of the first campaign for dataset I are presented in an alternative way to also evaluate the effect of the network cardinality. Figs. 2 and 3 show the average number of activated links versus the total number of links in the network for $\gamma$ set to $-3 \mathrm{~dB}$ and $3 \mathrm{~dB}$, respectively. Again, the major observation is that all IC schemes clearly outperform the baseline and in particular SIC yields impressive performance in the low SINR domain, activating up to two times more links. As the number of links in the network increases, the performance of all schemes improves, due to the diversity, almost linearly but with very small slope. For low $\gamma$ and small networks of around 5 links, nearly all of them are activated with SIC. On the other hand, PIC and SLIC have a consistent absolute gain over SUD, activating one to two links more. For high $\gamma$, all IC schemes result in the activation of one to two links more than the baseline, so most of the gain is reaped by SLIC.

In the second simulation study, the performance of the general LA-SIC problem, under individual SINR thresholds, is evaluated using dataset I. Each link assumes, with equal probability, one of the SINR thresholds from the set $\{-3,0,3\} \mathrm{dB}$ and uses as activation weight the corresponding Shannon rate per bandwidth unit, in bits per second per Hertz. The formulation (4) is implemented varying the maximum number of cancellation stages $T_{k}=T, \forall k \in \mathcal{K}$, from 0 , corresponding to the baseline case without IC, up to 3 . Fig. 4 shows the average spectral efficiency of the activated links versus the network cardinality. A roughly linear increase with similar slopes is experienced for both SUD and SIC. The increase however saturates for networks of more than 25 links. The efficiency is almost doubled with SIC; roughly half of this increase is achieved by the first cancellation stage and most of the rest by the next stage. Beyond the second cancellation the gains are diminishing.

The optimal solutions presented in this Section were found by an off-the-shelf solver, implementing standard techniques 


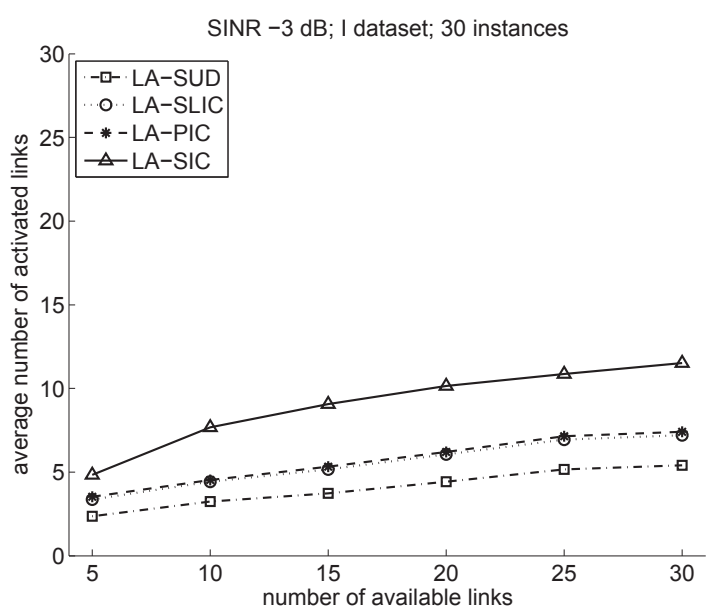

Fig. 2. Average number of activated links versus network size for SINR threshold $-3 \mathrm{~dB}$

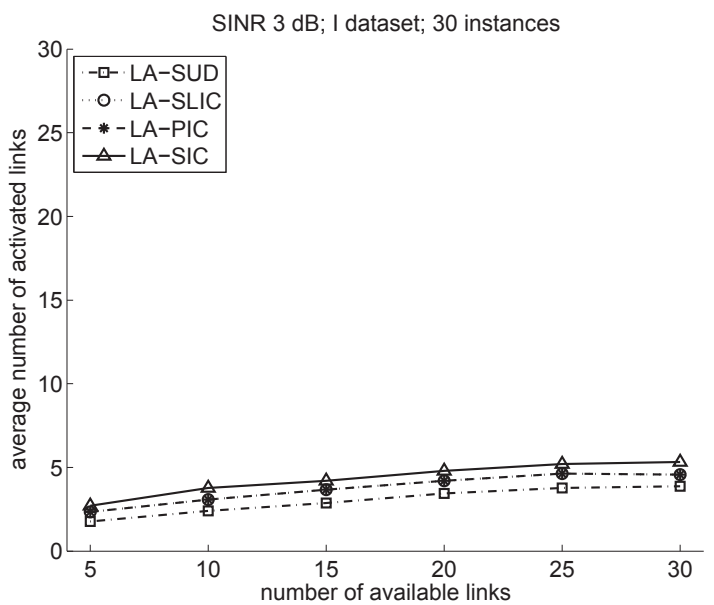

Fig. 3. Average number of activated links versus network size for SINR threshold $3 \mathrm{~dB}$

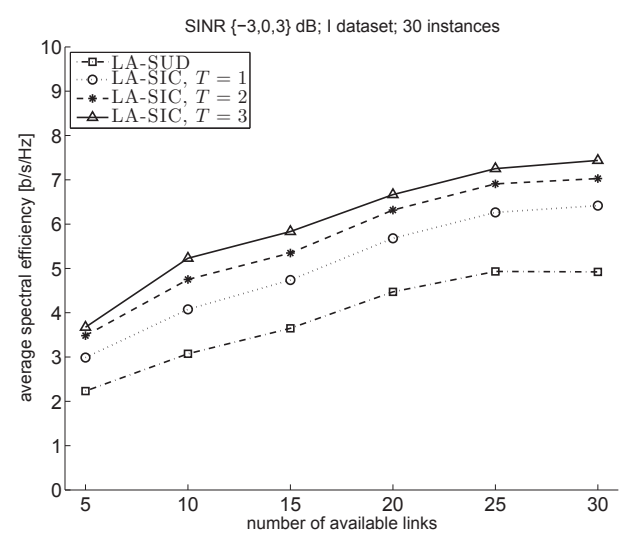

Fig. 4. Average spectral efficiency versus network size for SINR thresholds uniformly chosen from $\{-3,0,3\} \mathrm{dB}$ such as branch-and-bound and cutting planes [3]. The simulations were performed on a server with a quad-core AMD Opteron processor at $2.6 \mathrm{GHz}$ and $7 \mathrm{~GB}$ of RAM. The ILP formulations were implemented in AMPL 10.1 using the Gurobi Optimizer ver. 3.0. Regarding the computational complexity of the proposed ILP formulations for IC, an empirical measure is the running time of the solution process. Figs. 5a and 5b show the average solution time of all LA schemes versus network size, for the datasets I and N, respectively. The results are from the second simulation study, where $T=3$ for SIC. We observe that complexity is not an obstacle to conduct experiments for evaluation purposes, for the practical instance sizes we examine. The complexity of SLIC and PIC scales mildly and becomes significantly larger than the baseline for networks of more than about 20 links, whereas for SIC it increases exponentially. It is noted that the link properties greatly affect the complexity of IC since the largest average value in dataset I (200s for SIC in 30 links) is two orders of magnitude larger than the corresponding one in dataset $\mathrm{N}$.

\section{CONCLUSIONS}

We have addressed optimal concurrent link activation in wireless systems with parallel and successive interference cancellation (IC). The NP-hardness of the problems is presented and proven. Integer linear programming formulations are developed to approach the exact optimum. The results indicate that for low to medium SINR thresholds, IC delivers a significant performance improvement. An extension of the work is to incorporate power control that will bring another design dimension which can yield additional gains. In addition, given the proven computational complexity, the development of approximation algorithms for special cases of link activation and scheduling with interference cancellation calls for further research.

\section{REFERENCES}

[1] M. Andrews and M. Dinitz, "Maximizing capacity in arbitrary wireless networks in the SINR model: complexity and game theory," Proc. IEEE INFOCOM, 2009

[2] V. Angelakis, L. Chen, and D. Yuan, "Optimal and collaborative rate selection for interference cancellation in wireless networks," IEEE Commun. Lett., vol. 15, no. 8, pp. 819-821, 2011.

[3] D. Bertsimas and J. N. Tsitsiklis, Introduction to Linear Optimization. (Athena Scientific), 1997.

[4] P. Björklund, P. Värbrand, and D. Yuan, "Resource optimization of spatial TDMA in ad hoc networks radio networks: a column generation approach," Proc. IEEE INFOCOM, pp. 818-824, 2003.

[5] S.A. Borbash and A. Ephremides, "The feasibility of matchings in a wireless network," IEEE Trans. Inf. Theory, vol. 52, no. 6, pp. 27492755, 2006.

[6] G. Brar, D. M. Blough, and P. Santi, "Computationally efficient scheduling with the physical interference model for throughput improvement in wireless mesh networks," Proc. ACM MobiCom, 2006.

[7] A. Capone, G. Carello, I. Filippini, S. Gualandi, and F. Malucelli, "Routing, scheduling and channel assignment in wireless mesh networks: optimization models and algorithms,", Ad Hoc Netw., vol. 8, pp. 545563, 2010. 


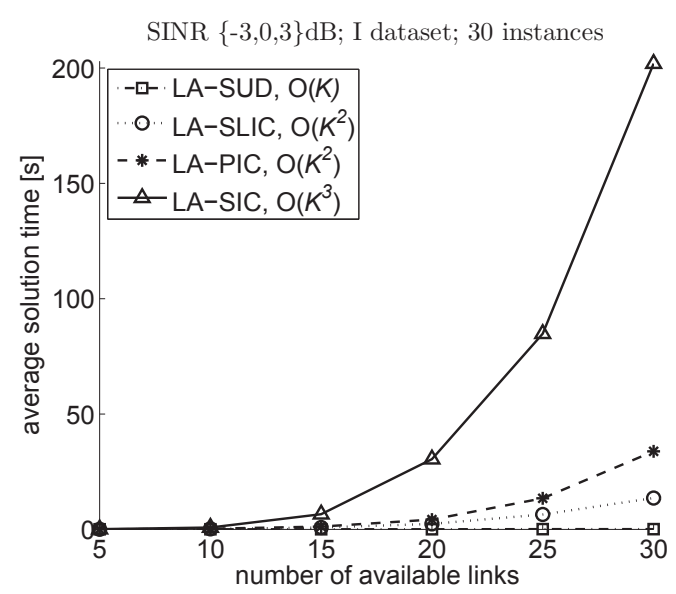

(a) I dataset

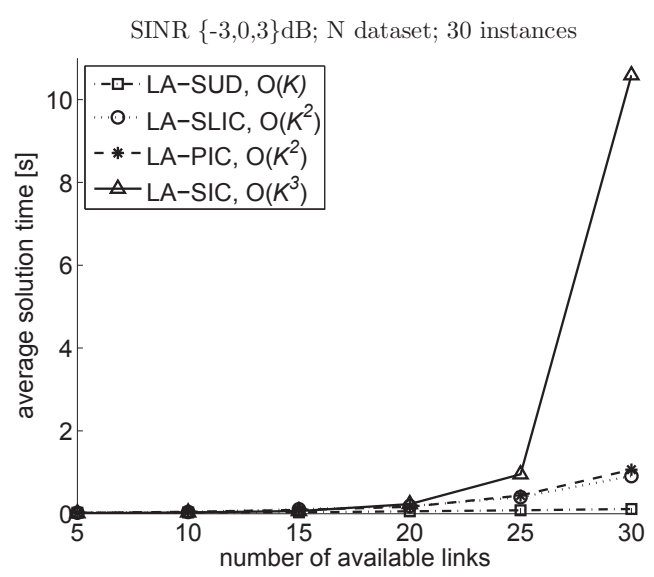

(b) $\mathrm{N}$ dataset

Fig. 5. Average solution time versus network size for SINR thresholds uniformly chosen from $\{-3,0,3\} \mathrm{dB}$

[8] A. Capone, L. Chen, S. Gualandi, and D. Yuan, "A new computational approach for maximum link activation in wireless networks under the SINR model," IEEE Trans. Wireless Commun., vol. 10, no. 5, pp. 13681372, 2011.

[9] J. El-Najjar, C. Assi, and B. Jaumard, "Joint routing and scheduling in WiMAX-based mesh networks," IEEE Trans. Wireless Commun., vol.9, no.7 pp. 2371-2381, 2010.

[10] L. Fu, S.C. Liew, and J. Huang, "Fast algorithms for joint power control and scheduling in wireless networks," IEEE Trans. Wireless Commun., vol.9, no.3, pp.1186-1197, 2010.

[11] O. Goussevskaia, Y. A. Pswald, and R. Wattenhofer, "Complexity in geometric SINR," Proc. ACM MobiHoc, 2007.

[12] P. Gupta and P. R. Kumar, "The capacity of wireless networks," IEEE Trans. Inf. Theory, vol. 46, pp. 388-404, 2000.

[13] J. Håstad, "Clique is hard to approximate within $n^{1-\epsilon}$, " Acta Mathematica, vol. 182, pp. 105-142, 1999.

[14] C. Jiang, Y. Shi, W. Lou, S. Kompella, and S. F. Midkiff, "Squeezing the most out of interference, an optimization framework for joint interference exploitation and avoidance," Proc. IEEE INFOCOM, 2012.

[15] E. Karipidis, D. Yuan, and E. G. Larsson, "Mixed-integer linear programming framework for max-min power control with single-stage interference cancellation," Proc. IEEE ICASSP, 2011.

[16] E. D. Lentz and J. Zhang, "Joint scheduling and interference cancellation in ad hoc networks," Proc. IEEE MILCOM, 2003.

[17] Y. Li and A. Ephremides, "A joint scheduling, power control, and routing algorithm for ad hoc wireless networks," Ad Hoc Netw., vol. 5, pp. 959 973, 2007.

[18] S. Lv, et al., "Scheduling in wireless ad hoc networks with successive interference cancellation," Proc. IEEE INFOCOM, 2011.

[19] A. Pantelidou, and A. Ephremides, "The scheduling problem in wireless networks," Journal of Communications and Networks, vol. 11, no. 5, 2009.

[20] S. Srinivasa and M. Haenggi, "Distance distributions in finite uniformly random networks: theory and applications," IEEE Trans. Vehicular Technol., vol. 59, no. 2, pp. 940-949, 2010.

[21] L. Tassiulas, and A. Ephremides, "Stability properties of constrained queueing systems and scheduling for maximum throughput in multihop radio networks," IEEE Trans. on Automat. Contr., vol. 37, no. 12, pp. 1936-1949, 1992.

[22] S. Verdu, Multiuser decoding, Cambridge, U.K.: Cambridge Univ. Press, 1998.

[23] C. Weeraddana, M. Codreanu, and M. Latva-aho, "On the advantages of using multiuser receivers in wireless ad-hoc networks," Proc. IEEE VTC 2009-Fall, 2009.

[24] X. Xu and S. Tang, "A constant approximation algorithm for link scheduling in arbitrary networks under physical interference model," Proc. ACM FOWANC, 2009. 